\title{
Demanda del servicio de atención farmacéutica en una farmacia comunitaria privada
}

\author{
Request of pharmaceutical care service in a private owned community pharmacy
} Cristiane Ferreira PIRES; Mauricélia Maria COSTA; Daniela ANGONESI; Felipe Pasquotto BORGES.

\begin{abstract}
RESUMEN *
El farmacéutico, además de productor de medicamentos, pasó a ser también co-responsable del tratamiento medicamentoso y promotor del uso racional de medicamentos. La aparición de una nueva filosofía, la atención farmacéutica, vino a estructurar, complementar y permitir ese nuevo papel del farmacéutico en la atención primaria de la salud.

Los objetivos del presente trabajo fueron determinar la existencia y caracterizar la demanda, verificar el conocimiento, interés y disposición al pago del servicio de atención farmacéutica en una farmacia comunitaria privada.

Se ha realizado a través de una entrevista guiada por check-list, como instrumento de recogida de datos, aplicado por entrevistadores. E verificó que de los 236 clientes entrevistados, el 88,1\% no conocían la expresión 'atención farmacéutica'; el $67,2 \%$ afirmaron tener interés por el servicio. En relación a la disposición al pago, el 39,9\% dijeron que dependía del valor, y el 10,1\% afirmó que pagaría por el servicio.

Esto nos permite concluir que existe demanda en este establecimiento, permitiendo evaluar la posibilidad de realización en otras poblaciones; llevándonos a desarrollar un modelo de practica en Brasil, o en el resto del mundo, para prestar atención primaria a la salud de los usuarios de medicamentos que la necesitan.
\end{abstract}

Palabras clave: Atención farmacéutica. Servicios de farmacia comunitaria. Demandas del paciente.

\begin{abstract}
Pharmacists, more than drug producer, is being a co-responsible for drug therapy and promoter of the rational use of medicines, enhancing their role. Appearance of a new philosophy, pharmaceutical care, came to organize, enhance and allowing this new role of the pharmacist in primary health care. Objectives of the present study were to determine the existence and to characterize the request for pharmaceutical care services and to assess the wiliness to pay for these services in a privately owned community pharmacy.

An interview following a check-list was used by researchers to gather data.

In 236 interviewed customers, $88.1 \%$ did not know the term 'pharmaceutical care', $67.2 \%$ showed to be interested on the service. Regarding the wiliness to pay, $39.9 \%$ conditioned it to the amount, and $10.1 \%$ stated that they would pay for the service. This allows us to conclude than in this setting, a demand existed, what allows repeating this survey in other settings, what lead us to the necessity of defining a standard of practice in Brazil, and in the rest of the world, to provide care to those who need it.
\end{abstract}

Keywords: Pharmaceutical care. Community pharmacy services. Patient's request.

\section{INTRODUCCIÓN}

El profesional farmacéutico, además de productor de medicamentos, asó a ser también un coresponsable del tratamiento medicamentoso y promotor del uso racional de medicamentos, adquiriendo mayor importancia en su papel. ${ }^{1} \mathrm{La}$ aparición de una nueva filosofía de ejercicio profesional, la atención farmacéutica, vino a estructurar, complementar y permitir este nuevo papel del farmacéutico en la atención sanitaria.

En el ámbito internacional y nacional, se han producido discusiones sobre esa practica profesional, en la búsqueda de la mejor comprensión de su significado, objetivando si adaptación e integración a los modelos de salud de cada país.
Farmacéutica comunitaria en Belo Horizonte (Brasil). Mauricélia Maria COSTA. Licenciada en Farmacia. Farmacéutica comunitaria en Belo Horizonte (Brasil). Daniela ANGONESI. Especialista en Salud Pública. Centro Universitário Newton Paiva, Belo Horizonte (Brasil).

Felipe Pasquotto BORGES. Master en Slud Pública. Agencia Nacional de Salud Suplementaria - Ministerio de Salud (Brasil). 
Ante este contexto, el Ministerio de Salud de Brasil creó un marco importante para el Sistema Único de Salud (SUS), y realizó a reorientación de la Asistencia Farmacéutica en el SUS con la aprobación de la Política Nacional de Medicamentos (PNM). ${ }^{2}$

En Brasil, el proceso de promoción del la atención farmacéutica se inició en el año 2000 mediante una consulta de experiencias y reflexiones sobre la atención farmacéutico en la web de la OPAS/OMS, que dio origen a la propuesta de Consenso Brasileño de Atención Farmacéutica. En ese documento la atención farmacéutica fue incorporada a la asistencia farmacéutica y, con ello, se consideró para la promoción y formación de profesionales para ese ejercicio, el modelo de atención y principios del SUS conforme a lo propuesto en el workshop y en las reuniones complementarias, $^{3}$ que de desarrollaron para integrar las acciones de salud.

La propuesta de consenso fue ampliamente divulgada y discutida en los siguientes eventos: workshop 2001, dos reuniones complementarias de 2002, en el I Foro Nacional de Atención Farmacéutica en 2003, en la i Conferencia Nacional de Política de Medicamentos y Asistencia Farmacéutica de 2003, y en el II Foro Nacional de Atención Farmacéutica de 2004, que contribuyeron con la elaboración de diversas estrategias y decisiones de responsabilidades. ${ }^{3-6}$

La elevada morbi-mortalidad relacionada con el uso de medicamentos, la evidencia de la posibilidad de la prevención de esa morbi-mortalidad, la posibilidad de reducción de costes en salud y la mejor calidad de vida del paciente que recibe atención farmacéutica, demostrada en anteriores estudios, ${ }^{7}$ motivó la realización del estudio de la demanda del servicio de atención farmacéutica.

Así, se pretende obtener el conocimiento sobre el interés de los clientes de una farmacia comunitaria privada y su disposición al pago por el servicio para apoyar la implantación de este servicio. De esta forma se espera que este trabajo contribuya a la expansión de esta práctica para la población que necesita cuidados farmacéuticos.

\section{MÉTODOS}

Se realizó un estudio descriptivo, transversal, empleando el método de estimación rápida a través de una entrevista orientada por un guión como instrumento de recogida de datos. Ese guión fue aplicado, de acuerdo con la disponibilidad de tiempo de los individuos, por entrevistadores entrenados, sobre una muestra de clientes que se dirigieron a los mostradores de solicitando algún producto farmacéutico en dos farmacias comunitarias privadas de Belo Horizonte-MG (Brasil).

Se realizó un ensayo piloto durante un día, para evaluar la aceptación de los clientes y la necesidad de alteraciones del instrumento de recogida de datos 0 en el método. La recogida de datos fue realizada de lunes a viernes, de 9 a 18 horas, en el periodo de 17 de marzo a 1 de abril de 2004.

De acuerdo con el instrumento de recogida de datos, la entrevista seguía la siguiente sistemática:

- Los clientes eran abordados e informados que estaba siendo realizada una investigación de opinión;

- El guión no era entregado a los clientes; las preguntas eran realizadas por los entrevistadores y en cuatro de las 10 preguntas se presentaban alternativas de respuesta.

- Cuando preguntaba el entrevistado por no entender el concepto de atención farmacéutica (la atención farmacéutica pretende prevenir, detectar y resolver problemas de salud relacionados con los medicamentos para mejorar la calidad de vida de los pacientes),se aclaraba que se realizaría un seguimiento por el que el farmacéutico aconsejaría sobre los problemas de salud y los medicamentos tratando de controlar el problema y mejorar su calidad de vida.

- Cuando los entrevistados planteaban dudas en el resto de las preguntas, se anotaba como 'ns' correspondiente a (no sabe).

Para la compilación de los datos, se desarrolló una base de datos en el programa Microsoft Excel 2000. A partir de esa base de datos se realizaron los análisis descriptivos con cálculos de medias y proporciones en el programa Statistical Package for Social Sciences (SPSS) versión 10.0.

\section{RESULTADOS}

En el estudio realizado, 241 clientes aceptaron participar, y otros 49 no aceptaron, de los que 5 iniciaron la entrevista y desistieron antes de terminar. De los 236 clientes que participaron íntegramente en la entrevista, el 66,6\% (150) eran mujeres, y presentaban una media de edad de 50,6 años, variando entre 18 y 87 años.

Cuando se preguntó a los entrevistados sobre el conocimiento de la expresión 'atención farmacéutica', se comprobó que el 11,9\% (28) la conocían y el $88,1 \%$ no la conocía. Estos últimos incluyen un $55,1 \%$ (113) de entrevistados que poseen educación superior o postgraduada, seguido de un $35,6 \%$ (73) con bachillerato. Estas proporciones no presentan diferencia significativa.

A partir del momento en que se orientaba a los entrevistados respecto de lo que sería la atención farmacéutica, el 67,2\% (158) afirmó que tenían interés por el servicio, seguido del $24,9 \%$ (60) que no presentaba interés, y un 7,1\% (17) que no sabía.

Analizando el interés de los clientes por el servicio de atención farmacéutica en relación al genero, de las 149 mujeres, el 73,2\% (109) demostraron interés por el servicio, mientras que la proporción de interesados entre los hombres fue de $57,0 \%$, según la tabla 1 (asociación estadisticamente significativa $p=0,031$ ). 


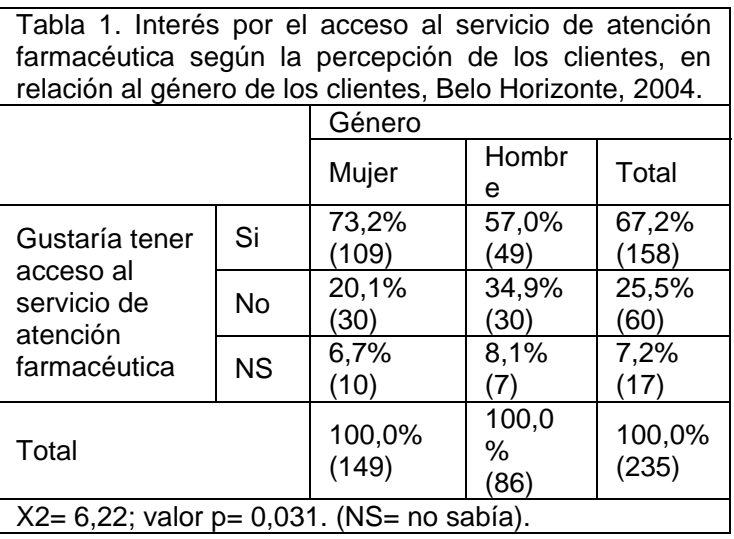

De las personas que afirmaron que tenían interés por el servicio después de la explicación del mismo (158), la mitad $(50,8 \%)$ de los entrevistados optó por no pagar (79), otro $39,9 \%$ (63) afirmaron que dependía del valor y un $10,1 \%$ (16) afirmaron que estarían dispuestos a pagar.

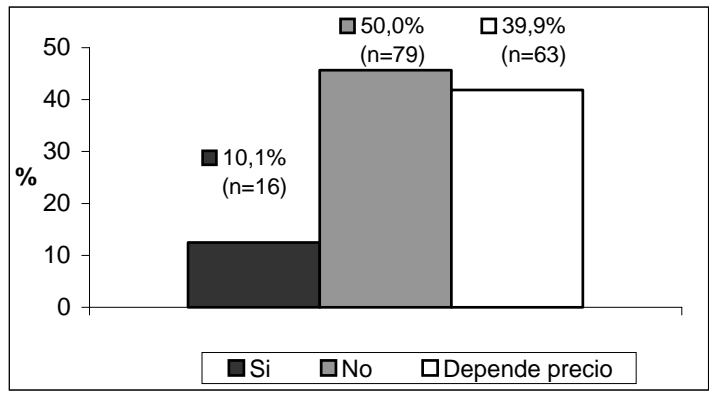

Figura 1: Disposición al pago por el servicio de atención farmacéutica, según la percepción de los clientes. Belo Horizonte, 2004.

Con relación a la intención de pagar por el servicio de atención farmacéutica, las alternativas más mencionadas fueron de hasta 5 reales ( 1,75 euros) y de 5 a 10 reales ( 1,75 a 3,5 euros), ambas con un $31,2 \%$ (5 clientes). De los que respondieron que dependía del precio a pagar, el 50,8\% (32) estaría dispuesto de pagar hasta 5 reales ( 1,75 euros) y el $38,1 \%$ (24)de 5 a 10 reales $(1,75$ a 3,5 euros) por visita en el periodo de seguimiento (tabla 2).

\section{DISCUSIÓN}

Los estudios sobre opinión de los clientes son de enorme importancia para el mejor desempeño de actividades en un establecimiento y la adecuación de las ofertas. Tratándose de un establecimiento de salud, se habla de satisfacción con los servicios sanitarios que tratan de mejorar la calidad de la dispensación, resolución de problemas, de solución de quejas, y de capacidad para satisfacer las necesidades del paciente.

El margen de edad de los entrevistados varió entre 18 y 87 años, lo que puede suponer una gran divergencia en opiniones sobre su salud. El perfil de escolaridad de los entrevistados apunta una de las limitaciones del trabajo, al no permitir comparaciones en torno a esa variable, ya que la mayoría de los entrevistados poseen estudios superiores o postgrados $(53,4 \% n=126)$, lo que ocurre en los barrios donde se realizó el trabajo en los que viven personas de clase media con alto nivel de escolaridad. ${ }^{8}$

De los 28 entrevistados que mencionan conocer la expresión 'atención farmacéutica', un número no cuantificado mencionó conocer ese servicio ofrecido por la Facultad de Farmacia de la Universidad Federal de Minas Gerais (UFMG), lo que demuestra la gran importancia de las actividades de atención a la salud desarrolladas por las universidades. La atención farmacéutica puede manifestarse en una amplia variedad de escenarios económicos y organizativos siendo el privado, individual $o$ en grupo, hasta el empleado en empresas, y desde el trabajo en ambulatorios hasta los cuidados intensivos. La principal meta de los estamentos sanitarios es hacer que la atención farmacéutica sea promovida y que la población tenga acceso a este servicio en la atención primaria, haciendo posible que aumente la demanda y añadiendo valor al establecimiento farmacéutico y al profesional.

En este estudio se puede observar que, al orientarlos sobre lo que sería la atención farmacéutica, la mayoría se mostró interesada por el servicio; mientras que los entrevistados que afirmaron que no se interesaban, lo justificaron por estar en seguimiento por profesionales médicos y por poseer seguros privados de salud que les facilita el acceso a estos profesionales, no necesitaban de otro profesional para su seguimiento. Se pudo verificar que el concepto de atención farmacéutica / seguimiento farmacoterapéutico, que intenta prevenir, detectar y resolver problemas de salud relacionados con los medicamentos para mejorar la calidad de vida de los pacientes, como fue diseñado por Hepler y Strand, ${ }^{1}$ y que fue utilizado en el guión de la entrevista, no se encuentra suficientemente claro para ser comprendido entre la población.

Tabla 2. Disposición a pagar por el servicio de atención farmacéutica de acuerdo con un valor hipotéticamente sugerido, según la percepción de los clientes, Belo Horizonte, 2004.

\begin{tabular}{|c|c|c|c|c|}
\hline & \multicolumn{3}{|c|}{$\begin{array}{l}\text { Pagaría por el servicio de } \\
\text { atención farmacéutica }\end{array}$} \\
\hline & & Si & $\begin{array}{l}\text { Depende } \\
\text { precio }\end{array}$ & Total \\
\hline \multirow{4}{*}{$\begin{array}{l}\text { Cuanto } \\
\text { pagaría } \\
\text { por el } \\
\text { servicio de } \\
\text { Atención } \\
\text { Farmacéuti } \\
\text { ca }\end{array}$} & $\begin{array}{l}<5 \\
\text { reales }\end{array}$ & $\begin{array}{c}31,2 \% \\
(5)\end{array}$ & $\begin{array}{c}50,8 \% \\
(32)\end{array}$ & $\begin{array}{c}46,8 \% \\
(37)\end{array}$ \\
\hline & $\begin{array}{l}5 \text { a } 10 \\
\text { reales }\end{array}$ & $\begin{array}{c}31,2 \% \\
(5)\end{array}$ & $\begin{array}{c}38,1 \% \\
(24)\end{array}$ & $\begin{array}{l}36,7 \% \\
(29)\end{array}$ \\
\hline & $\begin{array}{l}11 \text { a } 15 \\
\text { reales }\end{array}$ & $\begin{array}{c}18,8 \% \\
(3)\end{array}$ & $\begin{array}{c}6,3 \% \\
(4)\end{array}$ & $\begin{array}{c}8,9 \% \\
(7)\end{array}$ \\
\hline & $\begin{array}{l}>16 \\
\text { reales }\end{array}$ & $\begin{array}{c}18,8 \% \\
(3)\end{array}$ & $\begin{array}{c}4,8 \% \\
(3)\end{array}$ & $\begin{array}{c}7,6 \% \\
(6)\end{array}$ \\
\hline \multicolumn{2}{|l|}{ Total } & $\begin{array}{c}100 \% \\
(16)\end{array}$ & $\begin{array}{c}100 \% \\
(63)\end{array}$ & $\begin{array}{c}100 \% \\
(79)\end{array}$ \\
\hline
\end{tabular}

En el contexto en que nos encontramos en la presentación de este servicio, es necesario promover esa nueva práctica de forma diferente a la empleada normalmente. La estrategia debe dirigirse al pago para la prevención de la morbilidad relacionada con medicamentos. Así, los usuarios pagarían por el servicio, mucho mas interesados en 
prevenir que en resolver los problemas actuales del uso de medicamentos, lo que permitiría agregar mayor reconocimiento al servicio prestado. ${ }^{1}$

Una evidencia que apunta positivamente a la promoción de la atención farmacéutica en el sector privado, es que en relación a los entrevistados que aceptaron pagar por el servicio, de los que el 50,0\% (79) se mostró dispuesto a pagar dependiendo del valor. En países desarrollados, el ejercicio de la atención farmacéutica se encuentra más estructurado, como en los Estados Unidos. ${ }^{7}$ Estos autores relatan que por el servicio de atención farmacéutica se cobra una media de 13,57 USD en la visita inicial, llegando a 11,85 USD en la ultima visita de seguimiento, variando con la edad y la complejidad del caso. A lo largo del periodo de estudio, los autores observaron mejoría en el estado de salud del paciente, resolviendo los problemas relacionados con medicamentos (PRM) y alcanzando los objetivos terapéuticos; los tratamientos pasaron a ser menos complejos, llegando a una reducción general en los costes directos en salud del paciente. Es relevante que el asunto se lleve también al sector público, ya que la inversión para ofrecer el servicio puede ser compensada con una disminución de los gastos totales en salud.

De esa forma, puede considerarse positiva la percepción de la población en relación, tanto a su interés por el acceso, como por la intención al pago por el servicio, aunque no tengan el conocimiento esencial. Esto nos permite creer que, cuando este bien estructurada y difundida, se puede evaluar la posibilidad de remuneración del profesional farmacéutico por el servicio prestado, después de traer las mejoras significativas en la calidad de vida del usuario de medicamentos.

\section{CONCLUSIÓN}

Él ejercicio de la atención farmacéutica vino a estructurar, complementar y permitir un nuevo papel del farmacéutico en la atención primaria de la salud, como co-responsable del tratamiento medicamentoso y promotor del uso racional de medicamentos, que mejora realmente la calida de vida y los resultados terapéuticos del usuario de medicamentos. Los esfuerzos para su implantación y organización en Brasil no deben medirse, sino incluidos a nuestra determinación de hacerla acontecer.

Ante e estudio realizado sobre la demanda por el servicio de atención farmacéutica, se puede concluir que existe demanda en los establecimientos investigados, habiendo posibilidad de realización en otras poblaciones, aunque estemos en un punto de evolución y desarrollo de esta filosofía de practica en Brasil y en el mundo. Es importante entender que hay espacio para ofrecer ese servicio, trabajando una necesidad de los usuarios desconocida por ellos mismos y no satisfecha por ningún otro profesional.

En este momento, falta comprender que el encargo profesional requiere que se entienda que la sociedad necesita de los farmacéuticos, permitiendo que sean reconocidos como profesionales de la salud.

Nesse momento, resta compreender que o encargo profissional requer $o$ entendimento de que a sociedade necessita dos farmacêuticos, permitindo que sejam reconhecidos como profissionais da saúde, da mesma forma que o estabelecimento comercial farmacêutico muitas vezes não é reconhecido como um local de saúde, e nossa missão é o nosso compromisso de satisfazer esta necessidade.

\section{Referencias}

1. Hepler CD. Strand LM. Opportunities and responsibilities in Pharmaceutical Care. Am J Hosp Pharm 1990; 47: 533-43

2. Brasil. Ministério da Saúde. Portaria 3.916, de 30 de outubro de 1998. Aprova a Política Nacional de Medicamentos. Diário oficial da República Federativa do Brasil, Poder Executivo, Brasília, DF, 10 dez. 1998. Seção 1, p. 18.

3. Organização Pan-Americana da Saúde; Organização Mundial da Saúde. Consenso Brasileiro de Atenção Farmacêutica: proposta. Brasília, 2002b. Disponível em: http://www.opas.org.br/medicamentos/docs/PropostaConsensoAtenfar.pdf (Acesso em: 14/03/2005)

4. Organização Pan-Americana da Saúde; Organização Mundial da Saúde. Atenção Farmacêutica no Brasil: trilhando caminhos: Relatório 2001-2002. Brasília, 2002a. 28p. Disponível em: http://www.opas.org.br/medicamentos/docs/RelatorioAtenfar20012002.pdf (Acesso em: 14/03/2005)

5. II Fórum Nacional De Atenção Farmacêutica, 2004, Florianópolis. Relatório Final. Brasília: OPAS/OMS; 2004. Disponível em: http://www.opas.org.br/medicamentos/docs/HSE_FAF_FPS_1204.pdf (Acesso em: 14/03/2005)

6. Conferência Nacional de Medicamentos e Assistência Farmacêutica, 2003, Brasília. Relatório final preliminar. Brasília: Ministério da Saúde; 2003. 67p.

7 Cipolle RJ, Strand LM, Morley PC, Frakes M. Resultados del ejercicio de la Atención Farmacéutica. Pharmaceutical Care España, 2000. n. 2 p. 94-106

8 Souza JB. Aspectos Demográficos e Sócio Econômicos. In: Perfil Histórico, Econômico e Estatístico da Regional Oeste. Belo Horizonte: Gabinete da Secretaria Municipal da Coordenação de Gestão Regional Oeste, 2004. cap 2. p.15 